\title{
HOMOPARENTALIDADE: O ESTADO DA INVESTIGAÇÃO E A PROCURA DE NORMALIZAÇÃO
}

\author{
Pedro Alexandre Costa ${ }^{2}$ \\ Henrique Pereira ${ }^{2}$ \\ Isabel Pereira Leal ${ }^{3}$
}

Resumo: As competências parentais de mulheres lésbicas e de homens gays têm sido contestadas com base em diversos argumentos assentes no pressuposto de que a sua orientação sexual implica uma menor capacidade parental. Esta contestação legal, política e social, parece no entanto não conhecer eco na comunidade científica. A quantidade de estudos dedicados à parentalidade homossexual tem crescido exponencialmente e revelado que a orientação sexual de pais e mães não influencia negativamente o desenvolvimento das crianças. Através de uma revisão teórica de estudos pretende-se situar o estado da investigação na área da homoparentalidade adoptando uma visão crítica dos principais paradigmas de investigação e da forma como estes influenciam as conclusões dos estudos. Conclui-se que a inexistência de diferenças entre famílias homoparentais e famílias heteroparentais é equívoca, e que os esforços de normalização e minimização de eventuais diferenças comprometem o conhecimento científico sobre estas famílias.

Palavras-chave: Parentalidade; pais gays; mães lésbicas; desenvolvimento infantil.

Same-sex Parenting: State of the art and search for normalization (Abstract): Parenting skills of lesbian women and gay men have been challenged based on a number of arguments that are based on the assumption that sexual orientation is an indicator of lesser parental capacity. Nevertheless, this legal, political, and social argument, does not seem to echo in the scientific community. The amount of studies dedicated to homosexual parenting have grown exponentially and consistently revealed that parents' sexual orientation does not negatively influence child development. Through a theoretical review of studies, it is intended to place the state of

\footnotetext{
${ }^{1}$ Unidade de Investigação em Psicologia e Saúde (UIPES), Instituto Superior de Psicologia Aplicada, pcosta@ispa.pt

${ }^{2}$ Universidade da Beira Interior

${ }^{3}$ Unidade de Investigação em Psicologia e Saúde (UIPES), Instituto Superior de Psicologia Aplicada
} 
research on parenting by homosexuals taking a critical view of the main research paradigms and how these influence the conclusions drawn from the studies. It is concluded that the inexistence of differences between homosexual and heterosexual families is misleading, and that efforts of normalization and minimization of differences undermines the scientific knowledge about these families.

Keywords: Parenting; gay fathers; lesbian mothers; child development.

\section{A diversidade de arranjos familiares}

Os arranjos familiares de mães lésbicas e de pais gays são muitos e diversos. Esta diversidade acontece face à dificuldade no acesso a formas de parentalidade biológica e adoptiva que, na maioria dos países ocidentais, são ainda quase exclusivas de famílias heteroparentais. Antes do fenómeno americano conhecido como lesbian baby boom ${ }^{4}$, a grande maioria das famílias homoparentais tratava-se de famílias reconstituídas após o coming-out (auto-identificação pública) do pai ou da mãe como homossexuais (Patterson, 1994; 2006). São as mudanças legais e sociais que têm vindo a ocorrer em diversos países ocidentais que têm tornado possível o acesso a diversas formas de parentalidade que estavam anteriormente restritas a casais de sexo diferente, ou a pessoas heterossexuais, como a adopção ou a inseminação artificial.

De facto, através do processo de Inseminação Artificial tornou-se possível mulheres lésbicas conceberem uma criança e educarem-na sozinhas ou numa relação conjugal, como é o caso de países como a Holanda ou a Bélgica (Bos \& Hakvoort, 2007; Brewaeys, 2001; Vanfraussen, Ponjaert-Kristffersen \& Brewaeys, 2002). Em países onde o acesso a esta técnica de reprodução medicamente assistida não é permitida fora de um casamento entre pessoas de sexo diferente, como é aliás o caso de Portugal, é no entanto possível fazê-lo de uma forma privada com recurso a doação de esperma. Não sendo esta via reprodutiva possível a homens, em situações onde a parentalidade biológica é fundamental, o recurso a uma 'barriga de aluguer' revela-se então uma via privilegiada (Bergman, Rubio, Green \& Padron, 2010). Através do recurso a inseminação artificial ou a uma 'barriga de aluguer', os arranjos parentais têm por sua vez múltiplas conjugações possíveis com base na negociação de envolvimento entre o dador de esperma, ou dadora de óvulos, e a família. De facto, estudos australianos têm revelado que em particular doadores homossexuais têm como principal motivação o

\footnotetext{
${ }^{4}$ Lesbian Baby Boom refere-se a um aumento significativo de mães lésbicas, especialmente por recurso a inseminação artificial.
} 
desejo de parentalidade, em muitos casos requerendo algum tipo de envolvimento com a criança (Riggs, 2008; Ripper, 2008).

Por outro lado, a adopção de crianças é uma realidade somente em países em que esta situação é legalmente possível para casais do mesmo sexo, ou a adopção singular, de uma forma mais ou menos explícita, por mulheres lésbicas e homens gays. Nos Estados Unidos, por exemplo, tem-se assistido a um aumento considerável do número de pessoas que recorrem a agências de adopção para concretizar o desejo de parentalidade, nomeadamente entre homens (Brodzinsky \& Evan B. Donaldson Adoption Institute, 2003; Downing, Richardson, Kinkler \& Goldberg, 2009; Erich, Hall, Kanenberg \& Case, 2009). Na Europa, o acesso à adopção por parte de casais do mesmo sexo ou pessoas gays e lésbicas singulares é possível, apenas, na Bélgica, Dinamarca, Islândia, Holanda, Noruega, Espanha, Suécia e Reino Unido (Commissioner for Human Rights, 2011) não havendo ainda nestes países estimativas nacionais ou investigação significativa com este tipo de famílias.

Nos países Europeus há diferenças consideráveis no que diz respeito às formas de parentalidade. Em países como a Holanda, Bélgica ou Alemanha, onde a legislação permite que as famílias recorram a diferentes vias como a adopção ou a inseminação artificial, são diversos os estudos com mães lésbicas que recorreram a inseminação artificial (Bos, van Balen \& van den Boom, 2005; Brewaeys, 2001; Herrmann-Green \& Gehring, 2007). Em contraste, em países em que não há um acesso a estas formas de parentalidade (como em Itália ou Portugal) ou em que este acesso é ainda recente (como é o caso de Espanha), a larga maioria das famílias homoparentais são ainda constituídas por filhos/as provenientes de relações heterossexuais anteriores (Costa, Pereira \& Leal, 2011a, 2011b; González \& López, 2009; Lelleri, Prati \& Pietrantoni, 2008).

Considerando esta situação, são ainda poucas as estimativas nacionais do número de famílias homoparentais. Estudos comunitários revelaram que em Itália o número de pessoas homossexuais com filhos/as varia entre $5 \%$ e 8\% (Lelleri et al. 2008) e em Portugal entre 3\% a 10\% (Costa et al., 2011a, 2011b), sendo a grande maioria destas crianças concebidas em relações heterossexuais anteriores. Devido ao facto de tanto a adopção como a reprodução medicamente assistida e a 'barriga de aluguer' estarem vetados a casais do mesmo sexo, torna-se ainda mais difícil chegar a números aproximados destas famílias, relevando-as para uma perigosa invisibilidade e desprotecção.

Em contraste, de acordo com o Censos americanos de 2000, estimava-se que mais de 60 mil casais de homens e mais de 90 mil casais de mulheres tinham filhos/as menores de 18 anos Foi também possível estimar o número de crianças em famílias homoparentais, sendo que 270 mil crianças cresciam em famílias com dois pais ou duas mães, e 540 mil com um pai gay ou uma 
mãe lésbica (US Census Bureau, 2003). Outras estimativas nacionais realizadas por meio de estudos comunitários revelaram que aproximadamente uma em cada cinco mulheres lésbicas eram mães e um em cada nove homens gays eram pais (Bryant \& Demian, 1994).

\section{A comparação com as famílias heteroparentais}

O primeiro impulso para o estudo das famílias homoparentais surge da preocupação com o desenvolvimento e bem-estar das crianças. Tanto nos Estados Unidos como na Europa têm sido diversos os casos de disputas de custódia de crianças cuja mãe ou pai se revelam homossexuais, colocando-se a questão de serem ou não capazes de assumir as funções parentais das crianças, e se a sua orientação sexual se reflectirá negativamente no desenvolvimento das mesmas. É aqui que a investigação científica ganha um papel fundamental do ponto de vista do impacto social.

De uma forma geral, os estudos dedicados às famílias homoparentais têm reportado a não existência de diferenças significativas entre crianças em famílias homoparentais e crianças em famílias heteroparentais no que diz respeito a diversos índices de desenvolvimento. Numa revisão de estudos publicados até ao ano de 2000, Anderssen, Amlie e Ytteroy (2002) reuniram 23 estudos empíricos de onde retiraram 7 categorias principais de desenvolvimento infantil: (1) desenvolvimento emocional; (2) preferências sexuais; (3) estigmatização; (4) comportamentos de género; (5) ajustamento comportamental; (6) identidade de género; e (7) funcionamento cognitivo. Após a análise dos resultados publicados nestes estudos, os autores concluíram não existirem diferenças significativas entre os dois grupos, ou problemáticas desenvolvimentais em crianças em famílias homoparentais. Resultados semelhantes foram reportados em outros estudos de meta-análise (Allen \& Burrel, 1996; Crowl, Ahn \& Baker, 2008).

Por outro lado, também os estudos dedicados às competências parentais e ajustamento relacional de pais gays e de mães lésbicas apontam, na sua generalidade, para a não existência de diferenças significativas quando comparados com pais e mães heterossexuais. No que diz respeito às características de pais gays e mães lésbicas não há qualquer indício de problemáticas ligadas à saúde mental ou à capacidade de estabelecer laços afectivos seguros com os/as seus/suas filhos/as (Bos et al., 2005; Patterson, 2006; Ryan, 2007), demonstrando-se também que casais do mesmo sexo e casais de sexo diferente com filhos/as apresentam níveis de comunicação e suporte conjugal semelhantes (Bos, van Balen \& van den Boom, 2004; Bos, van Balen \& van den Boom, 2007). 
Se os estudos comparativos e transversais apontam para a inexistência de diferenças assinaláveis entre famílias homoparentais e famílias heteroparentais, os estudos longitudinais com as primeiras não só reforçam as conclusões anteriores, como contribuem para a expansão do conhecimento sobre o desenvolvimento destas famílias, em especial no que diz respeito às consequências a médio e a longo prazo de crescer em famílias homoparentais. Nanette Gartrell e os seus colaboradores (Gartrell, Banks, Hamilton, Reed, Bishop \& Rodas, 1999; Gartrell, Banks, Reed, Hamilton, Rodas \& Deck, 2000; Gartrell, Deck, Rodas, Peyser \& Banks, 2005; Gartrell, Hamilton, Banks, Mosbacher, Reed, Sparks \& Bishop, 1996) são os responsáveis pelo primeiro e mais significativo estudo longitudinal, de origem norte-americana, com um desenho experimental de 25 anos que inicia aquando da inseminação artificial de cerca de 100 mulheres. Desde as primeiras entrevistas às futuras mães foi possível assinalar o forte desejo de parentalidade e planeamento de uma criança na família. Nos diferentes tempos de investigação, as mães reportaram elevados níveis de saúde tanto própria como das crianças, redes de suporte social de qualidade fora da família e um ajustamento comportamental dos/as filhos/as semelhante à população normativa. No entanto, não obstante a maioria das crianças terem uma atitude positiva em relação a terem duas mães, $18 \%$ das crianças experimentaram discriminação e bullying homofóbico na escola aos 5 anos, e $43 \%$ confessaram terem sido vítimas de algum tipo de discriminação com base na orientação sexual da(s) sua(s) mãe(s) aos 10 anos de idade (Gartrell et al., 1999; Gartrell et al., 2000; Gartrell et al., 2005; Gartrell et al., 1996).

Também em estudos com adolescentes e jovens adultos/as foram encontradas poucas ou nenhumas diferenças entre estes/as e adolescentes de famílias heteroparentais ao nível da auto-estima e bem-estar (Golombok, Tasker \& Murray 1997; Huggins, 1989; O’Connell, 1993; Tasker \& Golombok, 1995), ajustamento psicológico (Rivers, Poteat \& Noret, 2008; Wainright, Russel \& Patterson, 2004), qualidade das relações de pares (Tasker \& Golombok, 1995; Rivers et al., 2008; Wainright \& Patterson, 2008) e orientação sexual (Bailey, Bobrow, Wolfe \& Mikach, 1995; Tasker \& Golombok, 1995).

\section{Diferenças e semelhanças entre famílias homoparentais e famí- lias heteroparentais}

Num artigo teórico sobre famílias homoparentais, Victoria Clarke (2002) identifica quatro principais paradigmas de investigação. Segundo esta autora, o primeiro paradigma sustenta a hipótese da ausência de diferenças entre famílias homoparentais e famílias heteroparentais. A grande maioria da investigação tem revelado que pais e mães homossexuais não divergem sig- 
nificativamente das restantes famílias em domínios psicológicos e comportamentais cruciais (Stacey \& Biblarz, 2001). Este paradigma surge da necessidade de confrontar a patologização de que estas famílias são vítimas e de reduzir a importância da orientação sexual (e mesmo da sexualidade) no desenvolvimento individual humano (Kitzinger, 1989), mas incorre no problema de um reforço do padrão heterossexista de família e na normalização e invisibilidade das famílias homoparentais, o que impede um conhecimento mais aprofundado das dinâmicas e percursos únicos destas famílias.

Um segundo paradigma, mais conservador, sustenta que as famílias homoparentais são diferentes e desviantes. Argumentam contra a parentalidade por pessoas gays e lésbicas com base na patologização destes/as, especialmente argumentando que os/as filhos/as destas famílias encontrarão problemáticas ao nível da construção da identidade de género, identidade sexual, e terão uma maior probabilidade de desenvolver uma orientação sexual homossexual (Clarke, 2002). Mais, tendem igualmente a estigmatizar todas as pessoas homossexuais, e não só aquelas que têm ou desejam ter filhos (ver, por exemplo, Cameron \& Cameron, 1996). No entanto, estes estudos apresentam várias falhas metodológicas assim como uma interpretação abusiva dos resultados, tendo mesmo conduzido à suspensão do primeiro autor da Associação de Psicólogos Americanos (APA) em 1983 e a uma repreensão por parte da Associação de Sociólogos Americanos (ASA) por manipulação de dados de investigação.

O terceiro paradigma surge como reacção ao primeiro, impulsionado pelos argumentos de feministas lésbicas que sustentam que a maternidade lésbica é essencialmente diferente e transformativa e não segue o padrão patriarcal. $\mathrm{O}$ discurso é muito politizado com o intuito de afirmar uma identidade lésbica (de onde advém a maternidade lésbica) diferente, e possuindo qualidades que a maternidade heterossexual não possui. Segundo Clarke (2002), o problema que se coloca é que este paradigma demonstra uma maior preocupação com a agenda política do que com a ciência experimental, adoptando com frequência um discurso construído 'de lésbicas para lésbicas'.

Finalmente, o paradigma que sustenta que as famílias homoparentais são diferentes em consequência da opressão social e homofobia de que são vítimas. De acordo com Stacey (1996, p. 135), "as crianças de pais gays [e mães lésbicas] são vicariantemente vítimas de homofobia e heterossexismo institucional violentos. Todas elas sofrem de consideráveis desvantagens económicas, legais e sociais impostas pelos seus pais [e mães], por vezes de forma ainda mais severa. Elas arriscam-se a perder um pai [ou mãe] ao simples capricho de um juiz". Deste paradigma advém uma diversidade de estudos que apresentam algumas diferenças entre famílias homoparentais e heteroparentais. A literatura tem revelado que apesar de não serem encontradas diferenças significativas na incidência de homossexualidade nem em pro- 
blemáticas ligadas à construção de género em filhos/as de pais gays ou de mães lésbicas, estas crianças e adolescentes demonstram uma maior lateralidade nos comportamentos de género, menor tipificação dos papéis de género, maior conforto com a sua identidade de género, maior questionamento sobre a sua orientação sexual e uma maior frequência de experiências amorosas com pessoas do mesmo sexo (Bos \& Sandfort, 2010; Gartrell, Bos \& Goldberg, 2010; Golombok, 2000; Green, Mandel, Hotvedt, Gray \& Smith, 1986; Tasker \& Golombok, 1997). Mais, não obstante a literatura revelar não haver diferenças no que diz respeito à qualidade da relação de pares, alguns estudos revelam que as crianças, e em particular adolescentes em famílias homoparentais, são com frequência vitimizados/as e/ou estigmatizados/as na escola em função do género parental (Gartrell et al., 2005; Robitaille \& Saint-Jacques, 2009). Digno de referência é que estas experiências parecem não influenciar a auto-estima ou as competências sociais destas, supondo-se que o seu impacto seja mitigado por factores protectores como a qualidade da relação parental, comunicação aberta sobre a orientação sexual do pai ou da mãe na família, e contacto com famílias semelhantes (Bos \& van Balen, 2008; Fairtlough, 2008).

Por outro lado, têm sido também reportadas algumas diferenças no que diz respeito ao ajustamento relacional e práticas parentais em famílias homoparentais. 'Mães sociais' (mães não biológicas que asseguram as funções parentais), envolvem-se mais nas tarefas domésticas e de cuidados infantis (Bos et al., 2007; Patterson, 2002), têm um maior desejo de parentalidade (Bos et al., 2007), e utilizam menos comportamentos parentais de imposição de limites e demonstrações de poder (Bos et al., 2004; Bos et al., 2007) do que pais heterossexuais. Mães biológicas lésbicas revelam uma maior satisfação com a sua companheira e possuem um maior desejo de parentalidade (Bos et $a l ., 2007)$ do que mães heterossexuais. No geral, as famílias constituídas por duas mães apresentam maiores níveis de satisfação conjugal e melhor qualidade de interacção mãe-criança do que famílias de um pai e uma mãe (Bos et al., 2004; Brewaeys, Ponjaert, van Hall \& Golombok, 1997; Flaks, Fisher, Masterpasqua \& Joseph, 1995; Golombok et al., 1997).

Os estudos qualitativos com famílias homoparentais, ainda em pequena quantidade, permitem aceder a estas diferenças de uma forma mais compreensiva. Ao questionar os pais e as mães sobre as suas experiências de parentalidade, dificuldades e obstáculos, torna-se visível que a gestão da opressão social e a antecipação de dificuldades ligadas à discriminação é uma constante nos seus discursos, o que implica uma gestão quotidiana do stress, tanto por parte dos pais e mães como por parte das crianças (Hash \& Cramer, 2003; Lubbe, 2006; Robitaille \& Saint-Jacques, 2009). De facto, "pais [e mães] heterossexuais não sentem as mesmas pressões ou necessidade de assumir com 'orgulho' a sua heterossexualidade" (Gabb, 2001, p. 347). 
A invisibilidade normativa da heterossexualidade implica a gestão de uma série de tarefas de conciliação entre as diferentes identidades, assim como uma desvantagem de poder e de status com que necessariamente as famílias constituídas por pessoas gays ou lésbicas se confrontam e que inevitavelmente traz consigo diferenças.

O cruzamento em que a literatura se encontra é precisamente na explicação para estas diferenças. Como foi demonstrado, alguns/as autores/as pretendem minimizar as diferenças em forma de normalização destas famílias, enquanto outros/as utilizam estas diferenças sob a forma de agenda política de contestação da norma heterossexual ou de patologização da parentalidade homossexual. O que parece claro é que há diferenças inegáveis mas que não significa que estas diferenças tenham uma tradução directa quer na qualidade quer nas problemáticas destas famílias.

\section{Principais críticas metodológicas}

De uma forma geral as principais críticas apontadas aos estudos sobre homoparentalidade dizem respeito aos processos de amostragem e aos métodos utilizados. Uma das principais críticas consistentemente apontadas tem sido a utilização de amostras auto-selectivas, recrutadas através de amostragens de tipo snowball e/ou em organizações LGBT ${ }^{5}$ (Tasker, 2005; Tasker \& Patterson, 2006). As famílias que se voluntariam para estes estudos poderão não ser famílias típicas ou representativas das famílias homoparentais, nomeadamente famílias recrutadas através associações ou redes LGBT. Estas famílias possuem à partida características menos comuns como o facto de estarem activamente envolvidas em organizações de defesa dos direitos das minorias sexuais, ou mesmo um elevado nível de conforto e de divulgação da sua configuração familiar. Este envolvimento pode também implicar uma maior aceitação por parte da família de origem e a presença de uma rede social de que a maioria das famílias poderá não gozar. O número de participantes é também, com frequência, muito pequeno o que implica uma maior cautela na interpretação e na generalização dos resultados.

Uma outra crítica importante é que os/as investigadores/as desta área não controlam de uma forma sistemática a sua reactividade interpessoal às famílias, isto é, a sua assunção de 'normalidade' (ou de 'desvio' em alguns casos) destas famílias não é controlada aquando da análise dos resultados obtidos (Tasker, 2005; Tasker \& Patterson, 2006). Na grande maioria dos estudos, o objectivo da investigação é claro tanto para os/as participantes como para os/as investigadores/as o que poderá originar enviesamentos por

${ }^{5}$ A sigla LGBT refere-se a Lésbicas, Gays, Bissexuais e Transgéneros 
desejabilidade social dos/as participantes ou com base nas crenças dos/as investigadores/as.

Em resposta a estas críticas, estudos recentes provenientes tanto dos Estados Unidos como da Europa vêm colmatar algumas das falhas apontadas aos processos de amostragem através de duas formas: (1) recrutamento de famílias em clínicas de reprodução medicamente assistida, e (2) análise de dados obtidos por meio de estudos nacionais representativos. Nos Estados Unidos e Reino Unido começam a ser publicados resultados sobre famílias homoparentais retirados de estudos nacionais representativos, o que permite ultrapassar o problema da auto-selecção de participantes (ver, por exemplo, Golombok, Perry, Burston, Murray, Mooney-Somers, Stevens \& Golding, 2003; Wainright et al., 2004). Por outro lado, em alguns países Europeus como é o caso da Alemanha, Bélgica ou Holanda, e também nos Estados Unidos, é já possível recolher amostras aleatórias e em número suficiente através de clínicas de reprodução medicamente assistida (Bos et al., 2004, 2007; Brewaeys et al., 1997; Chan, Raboy \& Patterson, 1998; Herrmann-Green, \& Gehring, 2007). Contudo, estes estudos são limitados a casais de lésbicas que recorrem a esta forma de concepção, não sendo por isso representativos da população.

Por outro lado, a diversidade de métodos utilizados no estudo de famílias homoparentais é advogado por vários investigadores/as como uma das principais mais-valias destes estudos. Não obstante a validade das críticas aqui referidas, o facto de os resultados serem semelhantes mesmo quando obtidos através de diferentes métodos e metodologias de investigação vem reforçar a consistência das suas conclusões. Os diferentes desenhos de investigação aqui referidos (transversais, comparativos, longitudinais e qualitativos) têm em todos os casos concluído a inexistência de problemáticas associadas à parentalidade por pais gays ou mães lésbicas em domínios fundamentais da saúde psicológica.

De acordo com Anderssen e seus colegas "devido aos resultados não ambíguos nos estudos revistos, acreditamos que estudos epidemiológicos maiores e com instrumentos e testes mais robustos são menos necessários do que métodos mais aprofundados e orientados para os processos" (Anderssen et al., 2002; p. 349). Por outras palavras, mais do que prosseguir uma linha de investigação que procura normalizar as famílias homoparentais minimizando as suas diferenças, seria aconselhável que os/as investigadores/as procurassem avaliar e compreender as necessidades e processos específicos destas famílias. 


\section{Conclusão e direcções futuras}

Segundo a Academia Americana de Pediatras (AAP), não há diferenças sistemáticas entre pais gays e mães lésbicas e pais e mães heterossexuais na saúde emocional, competências parentais e atitudes acerca da parentalidade. Nenhum estudo revelou qualquer risco para as crianças como resultado de crescerem numa família com um ou mais pais gays ou mães lésbicas (Perrin \& Committee on Psychosocial Aspects of Child and Family Health, 2002). Semelhantes pareceres foram publicados pelas principais sociedades científicas norte-americanas, como é também o caso da Associação de Psicólogos Americanos (Paige, 2005). Os estudos comparativos entre famílias homoparentais e famílias heteroparentais foram necessários no sentido de proteger as primeiras e de salvaguardar os interessas das crianças que se encontravam à guarda de mães lésbicas ou de pais gays. Estes estudos foram impulsionados pelo número crescente de disputas parentais após o coming-out do pai ou mãe como homossexual nos Estados Unidos, embora actualmente seja já seguro afirmar que as crianças e adolescentes com pais ou mães lésbicas terão um desenvolvimento psicológico, emocional e social dentro dos parâmetros normativos. Contudo, como foi aqui demonstrado, a prossecução desta linha de investigação pode ser contraproducente para o conhecimento científico ao minimizar as diferenças existentes. As famílias homoparentais confrontam-se com desafios únicos que é necessário compreender e avaliar, especialmente quando se observa que as crianças e adolescentes poderão estar em maior risco de serem vitimizados/as ao longo da sua vida com base na configuração familiar. Desta forma, importa conhecer as situações e características destes episódios de forma a poder introduzir mudança nos contextos em que estas acontecem. Mais, pouco se sabe dos processos que permitem às crianças com uma maior incidência de vitimização demonstrarem elevados níveis de saúde mental, ajustamento comportamental e auto-estima, colocando-se a hipótese de que a vitimização anterior dos pais ou mães lhes permite estarem preparados/as para antecipar as dificuldades e estimular comportamentos resilientes nas crianças (Stacey \& Biblarz, 2001).

Mais recentemente é também sugerido que a orientação sexual dos pais e mães per se não tem consequências nem ao nível do desenvolvimento infantil nem no ajustamento e qualidade relacional de casais do mesmo sexo, embora o género dos pais e mães possa estar associado à aprendizagem dos papéis e comportamentos de género das crianças, especialmente no caso de casais de lésbicas. $\mathrm{O}$ género dos pais, ou mesmo a interacção entre género e orientação sexual, está envolvido na criação de novos tipos de estruturas e processos familiares que importa conhecer (Stacey \& Biblarz, 2001). Asso- 
ciado a estas questões ainda sem resposta está também o vazio na literatura dedicada a pais gays. Se por um lado são conhecidos vários estudos com mães lésbicas que recorrem às novas tecnologias de reprodução, poucos estudos conseguiram recolher amostras de pais gays em situações semelhantes ainda que se verifique já o fenómeno gay baby boom cujos pais recorrem a adopção ou 'barrigas de aluguer' como via de parentalidade. Partindo do pressuposto que é o género dos pais e mães que pode estar na génese das diferenças, é especialmente importante aprofundar o conhecimento sobre a parentalidade de homens gays.

\section{Referências}

Allen, M., \& Burrell, N (1996). Comparing the impact of homosexual and heterosexual parents on children: meta-analysis of existing research. Journal of Homosexuality, 32, 19-35.

Anderssen, N., Amlie, C., \& Ytteroy, E. A. (2002). Outcomes for children with lesbian or gay parents: a review of studies from 1978 to 2000. Scandinavian Journal of Psychology, 43, 335-351.

Bailey, J. M., Bobrow, D., Wolfe, M., \& Mikach, S. (1995). Sexual orientation of adult sons of gay fathers. Developmental Psychology, 31, 124-129.

Bergman, K., Rubio. R. J., Green, R., \& Padron, E. (2010). Gay men who become fathers via surrogacy: the transition to parenthood. Journal of GLBT Family Studies, 6, 111-141.

Bos, H. M. W., \& Hakvoort, E. M. (2007). Child adjustment and parenting in planned lesbian families with known and as-yet unknown donors. Journal of Psychosomatic Obstetrics \& Gynecology, 28, 121-129.

Bos, H., \& Sandfort, T. G. M. (2010). Children's gender identity in lesbian and heterosexual two-parent families. Sex Roles, 62, 114-126.

Bos, H. M. W., \& van Balen, F. (2008). Children in planned lesbian families: Stigmatization, psychological adjustment and protective factors. Culture, Health \& Sexuality, 10, 221-236.

Bos, H. M. W., van Balen, F., \& van den Boom, D. (2004). Experience of parenthood, couple relationship, social support, and child-rearing goals in planned lesbian mother families. Journal of Child Psychology and Psychiatry, 45, 755-764.

Bos, H. M. W., van Balen, F., \& van den Boom, D. C. (2005). Lesbian families and family functioning: an overview. Patient Education and Counseling, 59, 263-275 .

Bos, H. M. W., van Balen, F., \& van den Boom, D. (2007). Child adjustment and parenting in planned lesbian-parent families. American Journal of Orthopsychiatry, 77, 38-48.

Brewaeys, A. (2001). Review: parent-child relationships and child development in donor insemination families. Human Reproduction Update, 7, 38-46. 
Brewaeys, A., Ponjaert, I., van Hall, E. V., \& Golombok, S. (1997). Donor insemination: child development and family functioning in lesbian mother families. Human Reproduction, 12, 1349-1359.

Brodzinsky, D. M., \& Evan B. Donaldson Adoption Institute (2003). Adoptions by lesbians and gays: A national survey of adoption agency policies, practices and attitudes. Acedido: Fevereiro 2011 em http://www.adoptioninstitute.org/ publications/Lesbian\%20and\%20Gay\%20Adoption\%20Report_final.pdf.

Bryant, A. S., \& Demian (1994). Relationship characteristics of American gay and lesbian couples: findings from a national survey. Journal of Gay \& Lesbian Social Services, $1,101-117$.

Cameron, P., \& Cameron, K. (1996). Homosexual parents. Adolescence, 31, $757-$ -776 .

Chan, R. W., Raboy, B., \& Patterson, C. J. (1998). Psychological adjustment among children conceived via donor insemination by lesbian and heterosexual mothers. Child Development, 69, 443-457.

Clarke, V. (2002). Sameness and difference in research on lesbian parenting. Journal of Community \& Applied Social Psychology, 12, 210-222.

Commissioner for Human Rights (2011, June). Discrimination on grounds of sexual orientation and gender identity in Europe. Strasbourg: Council of Europe Publishing.

Costa, P. A., Pereira, H., \& Leal, I. (2011a, Julho). Desenvolvimento da escala revista de ajustamento diádico (RDAS) com casais do mesmo sexo. Sessão de posters apresentados no VIII Congresso Iberoamericano / XV Conferência Internacional de Avaliação Psicológica, Lisboa.

Costa, P. A., Pereira, H., \& Leal, I. (2011b, Julho). Validade retrospectiva da RDAS em relacionamentos entre pessoas do mesmo sexo. Sessão de posters apresentados no VIII Congresso Iberoamericano / XV Conferência Internacional de Avaliação Psicológica, Lisboa.Crowl, A., Ahn, S., \& Baker, J. (2008). A meta-analysis of developmental outcomes for children of same-sex and heterosexual parents. Journal of GLBT Family Studies, 4, 385-407.

Downing, J., Richardson, H., Kinkler, L., \& Goldberg, A. (2009). Making the decision: factors influencing gay men's choice of an adoption path. Adoption Quarterly, 12, 247-271.

Erich, S., Hall, S. K., Kanenberg, H., \& Case, K. (2009). Early and late stage adolescence: Adopted adolescents' attachment to their heterosexual and lesbian/gay parents. Adoption Quarterly, 12, 152-170.

Fairtlough, A. (2008). Growing up with a lesbian or gay parent: young people's perspectives. Health and Social Care in the Community, 16, 521-528.

Flaks, D., Ficher, I., Masterpasqua, F., \& Joseph, G. (1995). Lesbians choosing motherhood: a comparative study of lesbian and heterosexual parents and their children. Developmental Psychology, 31, 104-114.

Gabb, J. (2001). Desirous subjects and parental identities: constructing a radical discourse on (lesbian) family sexuality. Sexualities, 4, 333-352. 
Gartrell, N., Banks, A., Hamilton, J., Reed, N., Bishop, H., \& Rodas, C. (1999). The national lesbian family study: 2.Interviews with mothers of toddlers. American Journal of Orthopsychiatry, 69, 362-369.

Gartrell, N., Banks, A., Reed, N., Hamilton, J., Rodas, C., \& Deck, M. (2000). The national lesbian family study: 3 . Interviews with mothers of five-year-olds. American Journal of Orthopsychiatry, 70, 542-548.

Gartrell, N. K., Bos, H. M. W., \& Goldberg, N. G. (2010). Adolescents of the U.S. national longitudinal lesbian family study: Sexual orientation, sexual behavior, and sexual risk exposure. Archives of Sexual Behavior (online). Acedido em Julho 2011: http://www.springerlink.com/content/d967883qp3255733/

Gartrell, N., Deck, A., Rodas, C., Peyser, H., \& Banks, A. (2005). The national lesbian family study: 4.Interviews with the 10-year-old children. American Journal of Orthopsychiatry, 75, 518-524.

Gartrell, N., Hamilton, J., Banks, A., Mosbacher, D., Reed, N., Sparks, C. H., \& Bishop, H. (1996). The national lesbian family study: 1.Interviews with prospective mothers. American Journal of Orthopsychiatry, 66, 272-281.

Golombok, S. (2000). Parenting: what really counts. London: Routledge.

Golombok, S., Perry, B., Burston, A., Murray, C., Mooney-Somers, J., Stevens, M., \& Golding, J. (2003). Children with lesbian parents: A community study. Developmental Psychology, 39, 20-33.

Golombok, S., Tasker, F. L., \& Murray, C. (1997). Children raised in fatherless families from infancy: family relationships and the socioemotional development of children of lesbian and single heterosexual mothers. Journal of Child Psychology and Psychiatry, 38, 783-791.

González, M. M., \& López, F. (2009), Relaciones familiares y vida cotidiana de niños e niñas que viven con madres lesbianas o padre gays. Cultura y Educación, 21, 417-428.

Green, R., Mandel, J. B., Hotvedt, M. E., Gray, J., \& Smith, L. (1986). Lesbian mothers and their children: a comparison with solo parent heterosexual mothers and their children. Archives of Sexual Behavior, 7, 175-181.

Hash, K. M., \& Cramer, E. P. (2003). Empowering gay and lesbian caregivers and uncovering their unique experiences through the use of qualitative methods. Journal of Gay and Lesbian Social Services, 15, 47-63

Herrmann-Green, L., \& Gehring, T. (2007). The German lesbian family study: Planning for parenthood via donor insemination. Journal of GLBT Family Studies, 3, 351-395.

Huggins, S. L. (1989). A comparative study of self-esteem of adolescent children of divorced lesbian mothers and divorced heterosexual mothers. In F. W. Bozett (Ed.), Homosexuality and the family. New York: Harrington Park Press.

Johnson, S. M., \& O'Connor, E. (2002). The Gay Baby Boom - The psychology of gay parenthood. New York: New York University Press.

Kitzinger, C. (1989). Liberal humanism as an ideology of social control: the regulation of lesbian identities. In K. Gergen \& J. Shotter (Eds.), Texts of identity. London: Sage. 
Lelleri, R., Prati, G., \& Pietrantoni, L. (2008). Omogenitorialità: I risultati di una ricerca italiana. Difesa Sociale, $4,71-84$.

Lubbe, C. (2008). The experiences of children growing up in lesbian-headed families in South Africa. Journal of GLBT Family Studies, 4, 325-359.

O'Connell, A. (1993). Voices from the heart: the developmental impact of a mother's lesbianism on her adolescent children. Smith College Studies in Social Work, 63, 281-299.

Paige, R. U. (2005). Sexual orientation, parents, \& children. Proceedings of the American Psychological Association, Incorporated. Minutes of the meeting of the Council of Representatives July 28 \& 30, 2004, Honolulu, HI. Acedido em Fevereiro 2011: http://www.apa.org/about/governance/council/policy/ parenting.aspx

Patterson, C. J. (1994). Lesbian and gay families. Current Directions in Psychological Science, 3, 62-64.

Patterson, C. J. (2002). Lesbian and gay parenthood. In Mark H. Bornstein (Ed.), Handbook of Parenting, vol. 3, Being and becoming a parent. New York: Lawrence Erlbaum Publishers.

Patterson, C. J. (2006). Children of lesbian and gay parents. Current Directions in Psychological Science, 15, 241-244.

Perrin, E. C., \& Committee on Psychosocial Aspects of Child and Family Health (2002). Technical report: coparent and second-parent adoption by same-sex parents. Pediatrics, 109, 341-344.

Riggs, D. (2008) Lesbian mothers, gay sperm donors, and community: ensuring the well-being of children and families. Health Sociology Review, 17, 226-234.

Ripper, M. (2008). Australian sperm donors: public image and private motives of gay, bisexual and heterosexual donors. Health Sociology Review, 17, 313-325.

Rivers, I., Poteat, V. P., \& Noret, N. (2008). Victimization, social support, and psychosocial functioning among children of same-sex and opposite-sex couples in the United Kingdom. Developmental Psychology, 44, 127-134.

Robitaille, C., \& Saint-Jacques, M.-C. (2009). Social stigma and the situation of young people in lesbian and gay stepfamilies. Journal of Homosexuality, 56, 421-442.

Ryan, S. (2007). Parent-child interaction styles between gay and lesbian parents and their adopted children. Journal of GLBT Family Studies, 3, 105-132.

Stacey, J. (1996). In the name of the family: rethinking family values in the postmodern age. Boston, MA: Beacon Press.

Stacey, J., \& Biblarz, T. J. (2001). (How) does the sexual orientation of parents matter? American Sociological Review, 66, 159-183.

Tasker, F. (2005). Lesbian mothers, gay fathers and their children: A review. American Journal of Orthopsychiatry, 65,203-215.

Tasker, F., \& Golombok, S. (1995). Adults raised as children in lesbian families. American Journal of Orthopsychiatry, 65, 203-215.

Tasker, F., \& Golombok, S. (1997). Growing up in a lesbian family. New York: Guilford Press. 
Tasker, F., \& Patterson, C. J. (2006). Research on Lesbian and Gay Parenting: retrospect and prospect. Journal of GLBT Family Studies, 3, 9-34.

US Census Bureau (2003). Married couple and unmarried-partner households: 2000-Census 2000 special reports. Acedido em Fevereiro 2011: http://www.census.gov/prod/2003pubs/censr-5.pdf

Vanfraussen, K., Ponjaert-Kristoffersen, I., \& Brewaeys, A. (2002). What does it mean for youngsters to grow up in a lesbian family created by means of donor insemination? Journal of Reproductive and Infant Psychology, 20, 237-252.

Wainright, J. L., \& Patterson, C. J. (2008). Peer relations among adolescents with female same-sex parents. Developmental Psychology, 44, 117-126.

Wainright, J. L., Russel, S. T., \& Patterson, C. J. (2004). Psychological adjustment, school outcomes, and romantic relationships of adolescents with same-sex parents. Child Development, 75, 1886-1989. 